\title{
BMJ Open Western Australian pregnancy cohort (Raine) Study: Generation 1
}

\author{
Manon L Dontje, ${ }^{1}$ Peter Eastwood, ${ }^{2}$ Leon Straker ${ }^{3}$
}

To cite: Dontje ML, Eastwood P, Straker L. Western Australian pregnancy cohort (Raine) Study: Generation 1. BMJ Open 2019;9:e026276. doi:10.1136/ bmjopen-2018-026276

- Prepublication history for this paper is available online. To view these files, please visit the journal online (http://dx.doi org/10.1136/bmjopen-2018026276).

Received 24 August 2018 Revised 4 March 2019 Accepted 5 April 2019

\section{Check for updates}

\section{Author(s) (or their} employer(s)) 2019. Re-use permitted under CC BY-NC. No commercial re-use. See rights and permissions. Published by BMJ.

${ }^{1}$ School of Population and Global Health, University of Western Australia, Perth, Western Australia, Australia

${ }^{2}$ Centre for Sleep Science, School of Human Sciences, The University of Western Australia, Perth, Western Australia, Australia

${ }^{3}$ School of Physiotherapy and Exercise Science, Curtin University, Perth, Western Australia, Australia

Correspondence to Dr Manon L Dontje; dr.m.I.dontje@gmail.com

\section{ABSTRACT}

Purpose The purpose of the Raine Study is to improve human health and well-being by studying the life-course of a cohort of Western Australians, based on a life-course conceptual framework that considers interactions between genetics, phenotypes, behaviours, the environment and developmental and social outcomes.

Participants Between May 1989 and November 1991, 2900 pregnant women were enrolled in the Raine Study in Perth, Western Australia. In total, 2730 women gave birth to 2868 children (Generation 2) between August 1989 and April 1992. The mothers and fathers of Generation 2 are referred to as Generation 1 of the Raine Study. In the most recent Generation 1 follow-up, 636 mothers and 462 fathers participated.

Findings to date Until the 26-year follow-up of Generation 1 the focus of research within the Raine Study was on outcomes in Generation 2, with information on the parents mainly being used to examine its influence on their children's outcomes. For example, recent findings showed that several characteristics of mothers, such as obesity, early mid-gestational weight gain and socioeconomic status were associated with non-alcoholic fatty liver disease, adiposity and cardiometabolic characteristics in offspring. Other findings showed that parents with back pain were more likely to have offspring who experienced back pain. Also, non-linear and dynamic relationships were found between maternal working hours and offspring overweight or obesity.

Future plans The Raine Study will continue to provide access to its dense longitudinal genetic, phenotypic, behavioural, environmental, developmental and social data to undertake studies with the ultimate goal of improving human health and well-being. Analyses of data from the recent Generation 1 year 26 follow-up are underway. Trial registration number ACTRN12617001599369

\section{INTRODUCTION}

The Western Australian Pregnancy Cohort (Raine) Study (www.rainestudy.org.au) started in 1989-1991 in Perth, Western Australia, as 'a randomised controlled trial (RCT) of the influence of serial fetal ultrasounds on birth outcomes', ' funded by the National Health and Medical Research Council of Australia. Out of this RCT, and with funding from the Raine Medical Research Foundation, a cohort was established to investigate the origins of health and disease. ${ }^{12}$ The purpose of the Raine Study has since evolved to improve
Strengths and limitations of this study

- The Raine Study is a prospective multigenerational observational study.

- An extensive range of genetic, phenotypic, behavioural, environmental and developmental factors have been collected at multiple time points.

- Overall, the Raine Study participants are representative of the general population in Western Australia.

- Weaknesses of all generations of the Raine Study include its homogeneity in regard to the ethnicity of its participants, the moderate sample size and the gradual decline in the number of participants.

- Important weaknesses of the Generation 1 cohort relate to the relative inconsistency of the data across the follow-ups, as not all variables were collected at every follow-up, to variability in respondents (mostly mothers, but also fathers, primary caregivers, secondary caregivers, etc), meaning that data of all mothers and fathers are not available at each follow-up.

human health and well-being by studying the life-course of a cohort of Western Australians', based on a life-course conceptual framework that considers the interactions between genetics, phenotypes, behaviours, the environment and developmental and social outcomes. ${ }^{3}$

\section{COHORT DESCRIPTION}

The Raine Study is a prospective multigenerational observational study. Between May 1989 and November 1991, 2900 pregnant women were enrolled in the RCT in Perth, Western Australia. Inclusion criteria included: 16-20 weeks pregnant, sufficient proficiency in English, attending the public antenatal clinic at King Edward Memorial Hospital or a nearby private practice clinic, expecting to deliver at the hospital and intending to remain in Western Australia. More detailed information about the recruitment of these pregnant women has been published elsewhere. ${ }^{4}$ In total, 2730 women gave birth to 2868 children between August 1989 and April 1992, including 120 twins, 6 triplets and 


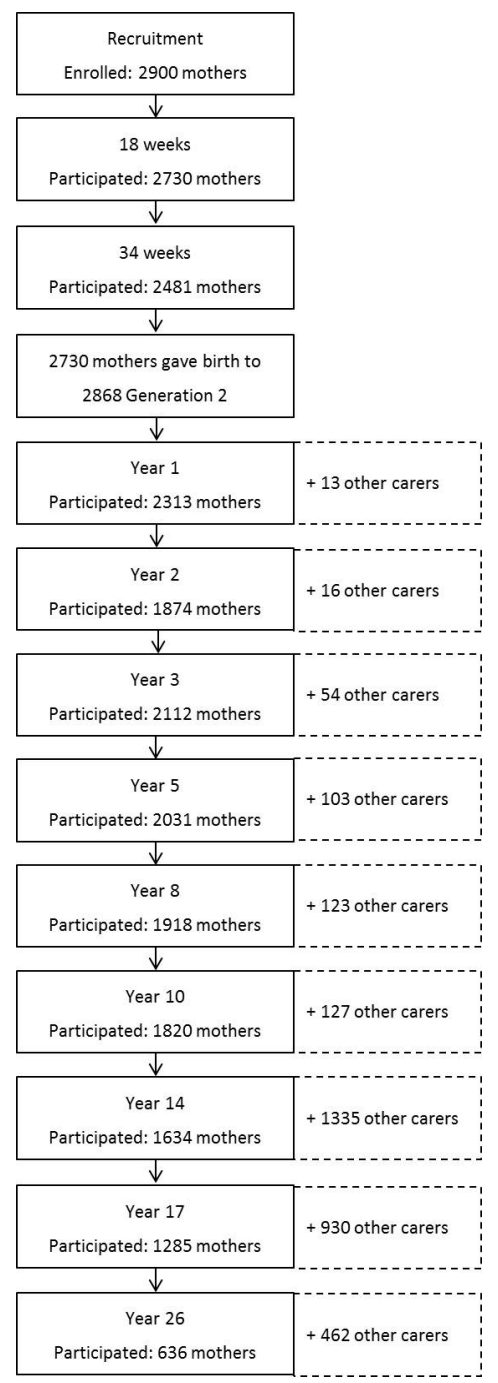

Figure 1 Flow diagram of Raine Study Generation 1 cohort participation (mothers only).

12 siblings (figure 1). All these children are the index participants (Generation 2) of the Raine Study and have been regularly followed up since birth. The mothers and fathers of Generation 2 are referred to as Generation 1 of the Raine Study and are the focus of this cohort profile paper. Detailed information of Generation 2 has been published elsewhere. ${ }^{3}$ The current cohort profile paper provides additional characteristics of Generation 1.

The year assigned to each follow-up relates to the age of the Generation 2 participants at the time of the data collection. Until the year 26 follow-up, the main focus of the Raine Study has been on Generation 2 and as a result, the data collection in the parents (Generation 1) has been less consistent and participation rates of Generation 1 are related to the participation rates of Generation 2. For example, since the beginning of the study until 2017 a total of 46 Generation 2 participants had died which automatically meant that their parents stopped participating in the study as well. Participation rates of Generation 1 have decreased somewhat over the years, as shown in figure 1 (see also Straker $e t a l^{3}$ ). In year 2, there was a dip in the participation rate which was caused by insufficient financial resources to complete the data collection of the whole cohort. In the earlier follow-ups, only mothers participated in the Generation 1 assessments, but later on some of the questionnaires were completed by fathers, grandparents, foster parents or step parents. In year 8 and year 10, the Generation 1 assessments were not specifically aimed at mothers anymore, but instead the 'primary caregiver' of the Generation 2 participant was asked to complete the questionnaires. Of those primary caregivers in year 8, 1918 were mothers, 71 fathers, 8 grandparents and 44 others (eg, foster mother, stepfather, not stated). In year 10, 1820 mothers participated, 89 fathers, 8 grandparents and 30 others (eg, foster mother, stepfather, not stated). In year 14 and year 17 , there were separate questionnaires and clinical assessments for primary and secondary caregivers. In year 14, 1634 mothers, 1162 fathers, 15 grandparents and 135 others (eg, foster mother, stepfather, not stated) participated. In year 17, 1285 mothers, 816 fathers, 10 grandparents and 104 others (eg, foster mother, stepfather, not stated) completed the questionnaires and assessments. Assessments at the year 26 follow-up included questionnaires and clinical assessments of both mothers $(n=636)$ and fathers $(n=462)$ of the Generation 2 participants.

\section{Representativeness of the generation 1 cohort}

The representativeness of the Raine Study Generation 1 compared with the Western Australian population has been examined at six time points: at the time of recruitment, at the time of birth of Generation 2, at the year 8 follow-up, at the year 14 follow-up, at the year 17 follow-up and at the year 26 follow-up. ${ }^{3}$

To examine the representativeness of the Raine Study Generation 1 cohort (mothers) at the time of recruitment, researchers kept records of all pregnant women attending the antenatal clinics during a 6 -month period halfway through the recruitment period. In this period, there were 131 clinic sessions attended by 1420 new women. Of these new attendees, $50 \%$ were eligible $(n=707)$ to participate. The primary reason for ineligibility was $>20$ weeks' gestation $(36 \%)$, followed by language difficulties $(8 \%)$, planned to deliver elsewhere $(4 \%)$ and psychosocial problems $(2 \%)$. During this audited period, the majority $(90 \%)$ of the eligible women agreed to participate $(\mathrm{n}=633){ }^{4}$

Data from the Western Australia Department of Health Midwives Notification System and Hospital Morbidity of all live births (excluding Raine Study births) in Western Australia during the 3-year recruitment period were used to examine the representativeness of the Raine Study Generation 1 (mothers) at the time of birth of Generation 2. Characteristics of the mothers (Generation 1) such as maternal age, marital status, ethnicity and arealevel socioeconomic status were investigated. The characteristics of the Raine Study mothers were similar to all Western Australian mothers, except that Raine Study 
mothers were slightly more often unmarried and firsttime mothers (table 1 in Straker $e t a \vec{l}^{3}$ ).

The representativeness of the Raine Study Generation 1 cohort was also examined at the year 8 follow-up, by comparing characteristics of the participants with the year 2001 Western Australian Population Census data. Overall, the Raine Study participants were representative of the general Western Australia population, except that more Raine Study parents were residing in Western Australia, were born overseas, had postsecondary and tertiary education, were working in clerical/retail occupations and had medium family income levels (see table 2 in Straker $\left.e t a l^{3}\right)$.

Also at the year 14 and year 17 follow-ups, the Raine Study Generation 1 cohort was representative of the general population in Western Australia. Cohort characteristics were compared with year 2006 Western Australian Population Census data of families living in Western Australia with children aged $15-17$ years. Overall, there were no substantial differences in most characteristics, except less Raine Study parents lived in rural areas, and more Raine Study parents had tertiary education. Also, in year 14 more Raine Study parents were in clerical/administrative occupations with medium-income levels, while in year 17 more Raine Study parents were in professional/ managerial occupations (see table 3 in Straker $e t a \vec{l}^{3}$ ).

To examine the representativeness of the Generation 1 cohort (mothers) at time of the year 26 follow-up, the study sample (mean (SD) age 55.6 (5.5) years) was compared with year 2016 Western Australian Population Census data of women aged 55-64years (table 1). Several demographic factors were compared, including marital status, place of birth, parity, level of education, occupation, family income, area-level socioeconomic status and indigenous status. Overall differences between the Raine Study Generation 1 cohort (mothers) and the general Western Australia population were small. Slightly more Raine Study Generation 1 participants (mothers) were married, born in Australia had three or more children, finished secondary school, had a non-school qualification at TAFE/college level or graduate/postgraduate level, had low and medium low incomes, had higher area-level socioeconomic status and were non-indigenous. Of the total Generation 1 cohort (mothers) that participated in the year 26 follow-up, $91.4 \%$ was Caucasian, $0.8 \%$ Aboriginal, $0.8 \%$ Polynesian, $0.3 \%$ Vietnamese, $3.0 \%$ Chinese, $3.0 \%$ Indian and $0.8 \%$ was classified as other.

To examine the potential existence of any attrition biases in the Generation 1 cohort, participants (mothers) and drop-outs (mothers) of each follow-up have been compared based on several characteristics of the mothers at the time of recruitment (table 2A,B). Differences in age, level of education, ethnicity, level of income and area-level socioeconomic status at the time of recruitment between participants and drop-outs were assessed and analysed using $\mathrm{X}^{2}$ tests for each follow-up. Although there were significant differences in the proportions of participants and drop-outs across all maternal characteristics,
Table 1 Comparison of Raine Study Generation 1 cohort (females only, $\mathrm{n}=636$, mean (SD) age 55.6 (5.5) years) at year 26 follow-up with contemporaneous Western Australian (WA) Census population (2016 Census data, females)

\begin{tabular}{|c|c|c|}
\hline & \multicolumn{2}{|c|}{ Year 26 follow-up } \\
\hline & $\begin{array}{l}\text { Raine Study } \\
(\%)\end{array}$ & WA (\%) \\
\hline \multicolumn{3}{|l|}{ Marital status ${ }^{\star}$} \\
\hline Married & 68.4 & 62.8 \\
\hline Separated & 5.7 & 4.5 \\
\hline Divorced & 16.5 & 19.4 \\
\hline Widowed & 2.8 & 5.5 \\
\hline Never married & 3.0 & 7.9 \\
\hline Missing & 0.2 & 0.0 \\
\hline \multicolumn{3}{|l|}{ Place of birth $†$} \\
\hline Australia & 58.5 & 54.0 \\
\hline Other & 41.5 & 38.6 \\
\hline Missing & 0.0 & 7.4 \\
\hline \multicolumn{3}{|l|}{ Parity (number of children ever born) $\ddagger$} \\
\hline 0 & 0.0 & 11.3 \\
\hline 1 & 9.4 & 10.4 \\
\hline 2 & 35.4 & 36.2 \\
\hline 3 & 31.9 & 22.8 \\
\hline 4 & 13.4 & 8.5 \\
\hline 5 & 4.7 & 2.5 \\
\hline 6 or more & 1.7 & 1.5 \\
\hline Missing & 3.5 & 6.9 \\
\hline \multicolumn{3}{|l|}{ Highest level of school education§ } \\
\hline Did not go to school & 0.3 & 0.8 \\
\hline Primary school & 0.6 & 3.0 \\
\hline Secondary school & 95.1 & 88.4 \\
\hline Missing & 3.9 & 7.9 \\
\hline \multicolumn{3}{|l|}{ Highest level of non-school education } \\
\hline TAFE, college level & 52.4 & 46.5 \\
\hline Undergraduate, bachelor degree level & 26.2 & 23.0 \\
\hline Graduate, postgraduate degree level & 21.4 & 11.2 \\
\hline Missing & 0.0 & 19.4 \\
\hline \multicolumn{3}{|l|}{ Occupation $^{\star *}$} \\
\hline Agriculture, forestry and fishing & 0.8 & 2.3 \\
\hline Mining & 0.9 & 1.3 \\
\hline Manufacturing & 0.6 & 3.0 \\
\hline $\begin{array}{l}\text { Electricity, gas, water and waste } \\
\text { services }\end{array}$ & 0.8 & 0.5 \\
\hline Construction & 2.0 & 2.7 \\
\hline Wholesale trade & 0.9 & 1.7 \\
\hline Retail trade & 5.5 & 9.9 \\
\hline Accommodation and food services & 2.4 & 3.9 \\
\hline Transport, postal and warehousing & 1.9 & 2.7 \\
\hline $\begin{array}{l}\text { Information media and } \\
\text { telecommunications }\end{array}$ & 0.8 & 0.6 \\
\hline Financial and insurance services & 1.6 & 2.3 \\
\hline Rental, hiring and real estate services & 1.4 & 2.1 \\
\hline
\end{tabular}


Table 1 Continued

\begin{tabular}{|c|c|c|}
\hline & \multicolumn{2}{|c|}{ Year 26 follow-up } \\
\hline & $\begin{array}{l}\text { Raine Study } \\
(\%)\end{array}$ & WA (\%) \\
\hline $\begin{array}{l}\text { Professional, scientific and technical } \\
\text { services }\end{array}$ & 2.8 & 4.7 \\
\hline Administrative and support services & 4.6 & 3.9 \\
\hline Public administration and safety & 1.1 & 7.4 \\
\hline Education and training & 15.1 & 17.8 \\
\hline Healthcare and social assistance & 23.4 & 25.5 \\
\hline Arts and recreation services & 1.6 & 1.3 \\
\hline Other services & 9.0 & 3.2 \\
\hline Missing & 22.8 & 3.2 \\
\hline \multicolumn{3}{|l|}{ Family income†† } \\
\hline Low & 16.7 & 10.0 \\
\hline Medium low & 19.3 & 16.7 \\
\hline Medium high & 24.5 & 25.0 \\
\hline High & 31.8 & 31.8 \\
\hline Missing & 7.7 & 14.6 \\
\hline \multicolumn{3}{|l|}{ Indigenous status $\ddagger \ddagger$} \\
\hline ATSI & 0.8 & 2.1 \\
\hline Non-indigenous & 99.2 & 91.8 \\
\hline Missing & 0.0 & 6.1 \\
\hline \multicolumn{3}{|l|}{ Area-level socioeconomic status§§ } \\
\hline 1 (most disadvantaged) & 7.2 & 10.6 \\
\hline 2 & 14.3 & 22.5 \\
\hline 3 & 16.8 & 18.8 \\
\hline 4 & 10.3 & 15.9 \\
\hline 5 (most advantaged) & 50.0 & 32.0 \\
\hline Missing & 1.4 & 0.2 \\
\hline
\end{tabular}

*Marital status: WA 2016 Census data age category 55-64 years. Raine data category 'married' includes de facto.

tPlace of birth: WA 2016 Census data age category 55-64 years. ‡Parity: WA 2016 Census data age category 55-59 years. §School education: WA 2016 Census data age category 55-64 years. INon-school education: highest completed non-school qualification, ie, educational attainments other than those of a pre-primary, primary, or secondary education. Excludes persons with schooling up to year 12 . Raine $n=458$, Census $n=93242$, age category $55-64$ years.

${ }^{\star *}$ Occupation: WA 2016 Census data age category 55-64 years. ††Family income: WA 2016 Census data based on weekly family income for single and couple parent families, Raine data based on total amount of households usual salary/wage, before tax, per week: low $<$ AUD\$799 per week; medium low AUD\$800-US\$1499; medium high AUD\$1500-US\$2499; high $\geq A U D \$ 2500$.

ł‡Indigenous status: WA 2016 Census data age category 55-59 years. $\S \S A$ rea-level socioeconomic status based on IRSAD, divided in quintiles (based on WA Census SEIFA 2016). Low levels=most disadvantaged, high levels=most advantaged. ATSI, Aboriginal or Torres Strait Islander; IRSAD, Index of Relative Socio-economic Advantage and Disadvantage; TAFE, technical and further education.

the generally small differences remained relatively stable across all follow-ups. In general, differences were small and in summary were limited to the level of education, income and area-level socioeconomic status. Specifically, over the years slightly fewer Generation 1 participants (mothers) had education to grade 10, low income and were in the low IRSAD tertile and slightly more participants had TAFE/trade education, high income and were in the high IRSAD tertile.

\section{Follow-ups}

To date, the Generation 1 cohort has participated in 12 follow-up studies. Each follow-up was approved by the institutional ethics committee and written informed consent from the participants was obtained for each follow-up. The mothers, fathers or other primary carers were involved in all of the follow-up studies of Generation 2 until that generation were adults. During these studies these parents answered questions about their children and about themselves. Several clinical assessments were also undertaken in the Generation 1 cohort, the first being when the mothers were around 18 weeks pregnant, followed by assessments when they were 34 weeks pregnant and when their child was born. Generation 1 assessments were also undertaken when their children (Generation 2) were $1,2,3,5,8,10,14$ and 17 years of age. Generation 1 has recently completed its first follow-up independently of Generation 2. The year assigned to this follow-up was year 26, as this was the age of the Generation 2 participants at the time of the study.

Until the year 8 follow-up, only mothers (and a few fathers, grandparents and foster parents) participated in the questionnaire and clinical assessments, except at the 18-week pregnancy follow-up when fathers also completed a short questionnaire. During these early follow-ups, mothers also provided information about the fathers of the Generation 2 participants. In the year 8 and year 10 follow-ups, questionnaires were completed by the primary caregiver, who provided information about the secondary caregiver as well. In year 14 and year 17 follow-ups, both primary and secondary caregivers participated in questionnaire and clinical assessments. Assessments at the year 26 follow-up included questionnaire and clinical assessments of both mothers and fathers of the Generation 2 participants.

\section{Data collection}

An extensive range of genetic, phenotypic, behavioural, environmental and developmental factors have been collected at multiple time points. A summary of the measurements at each follow-up is shown in table 3. Most questionnaires were self-completed by the participants, blood samples were collected by a trained phlebotomist and clinical measurements were taken by a trained research assistant. If participants were not comfortable or willing to answer specific questions, partake in specific tests or to give a blood sample, they were always given the option to opt out for that specific part.

Although Generation 2 data have been linked to a few administrative databases already, including educational records, there has not been any data linkage carried out with Generation 1 data yet. As it can be very useful for researchers to link Generation 1 data to certain administrative databases such as death registrations, cancer 


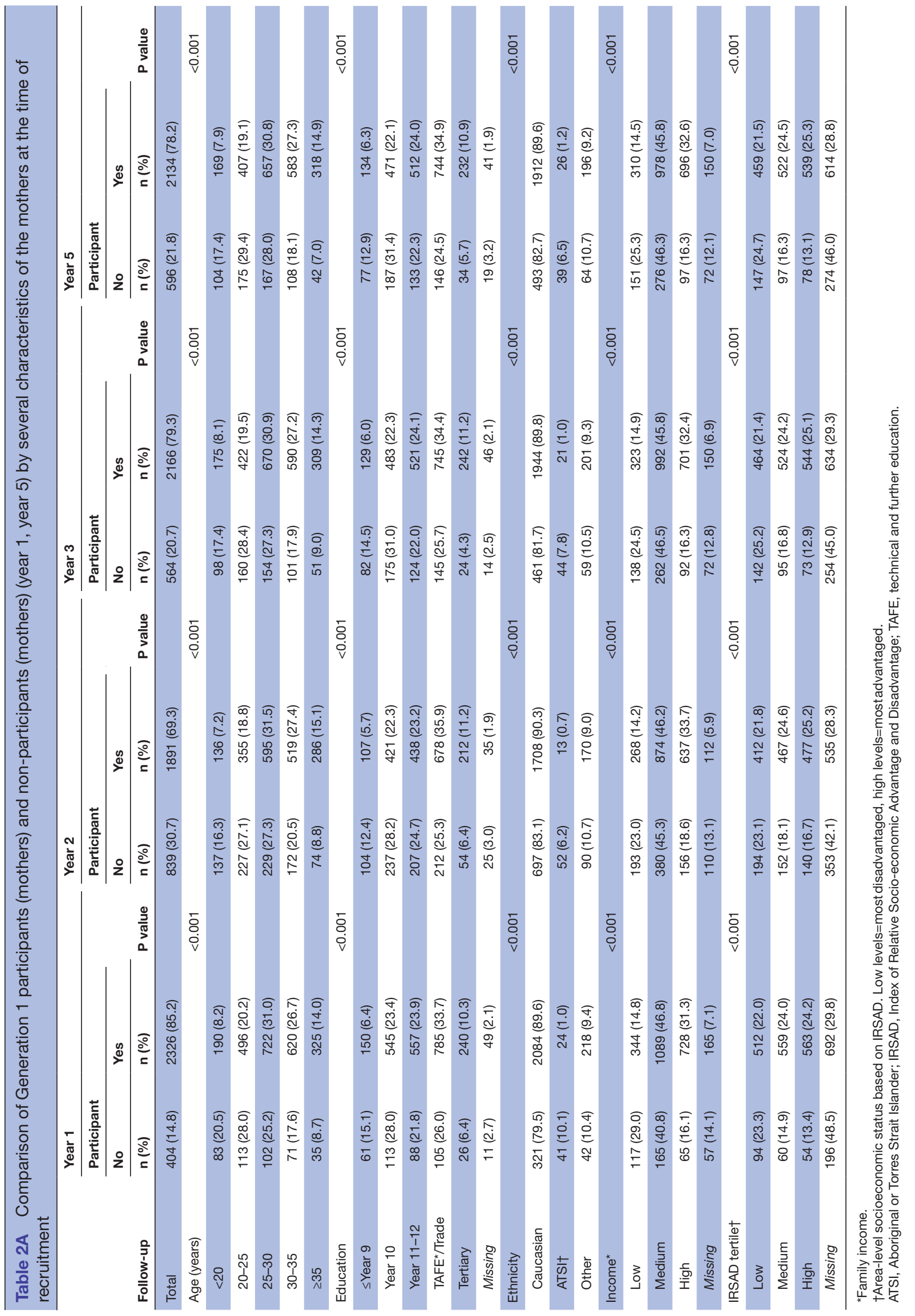




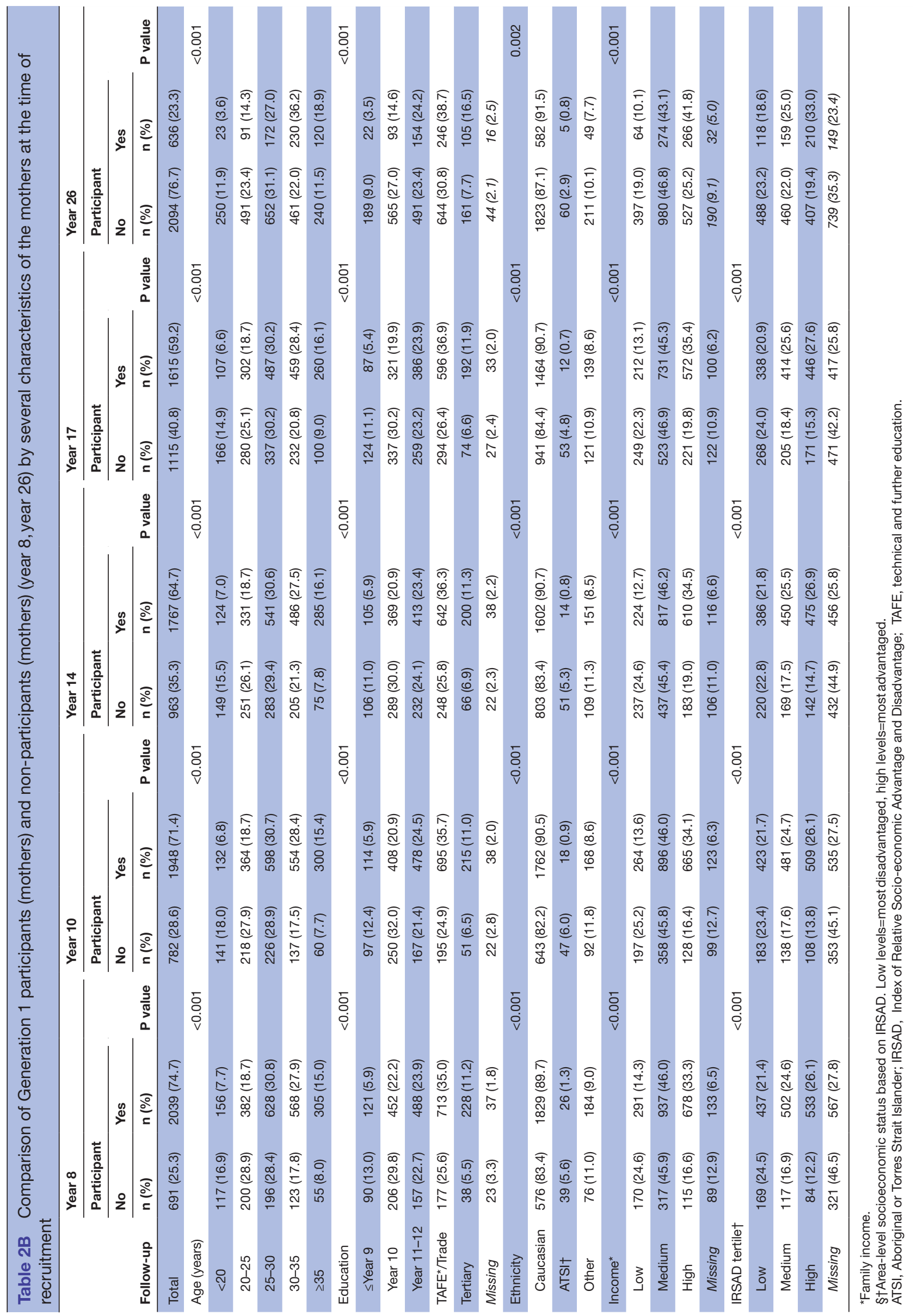




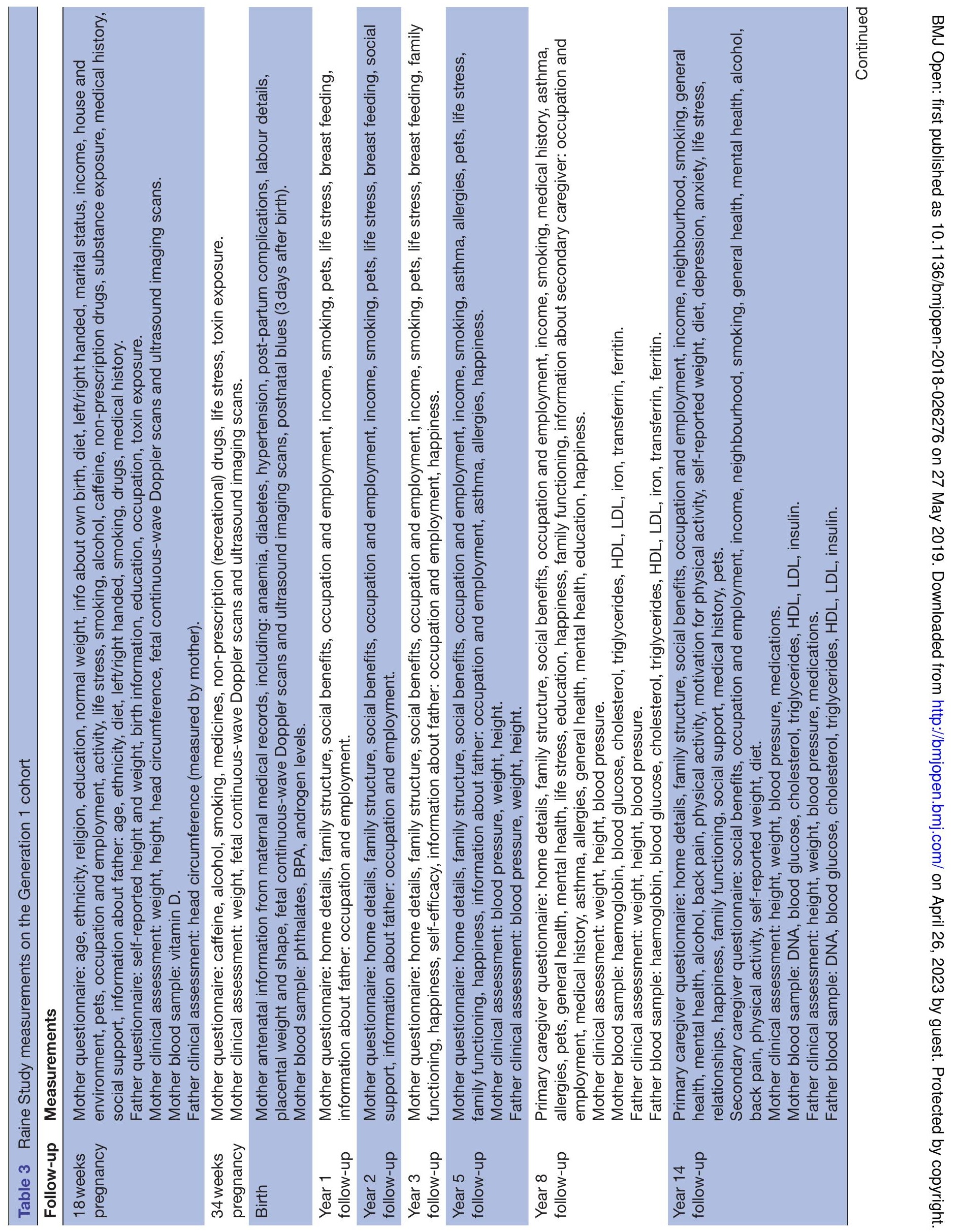




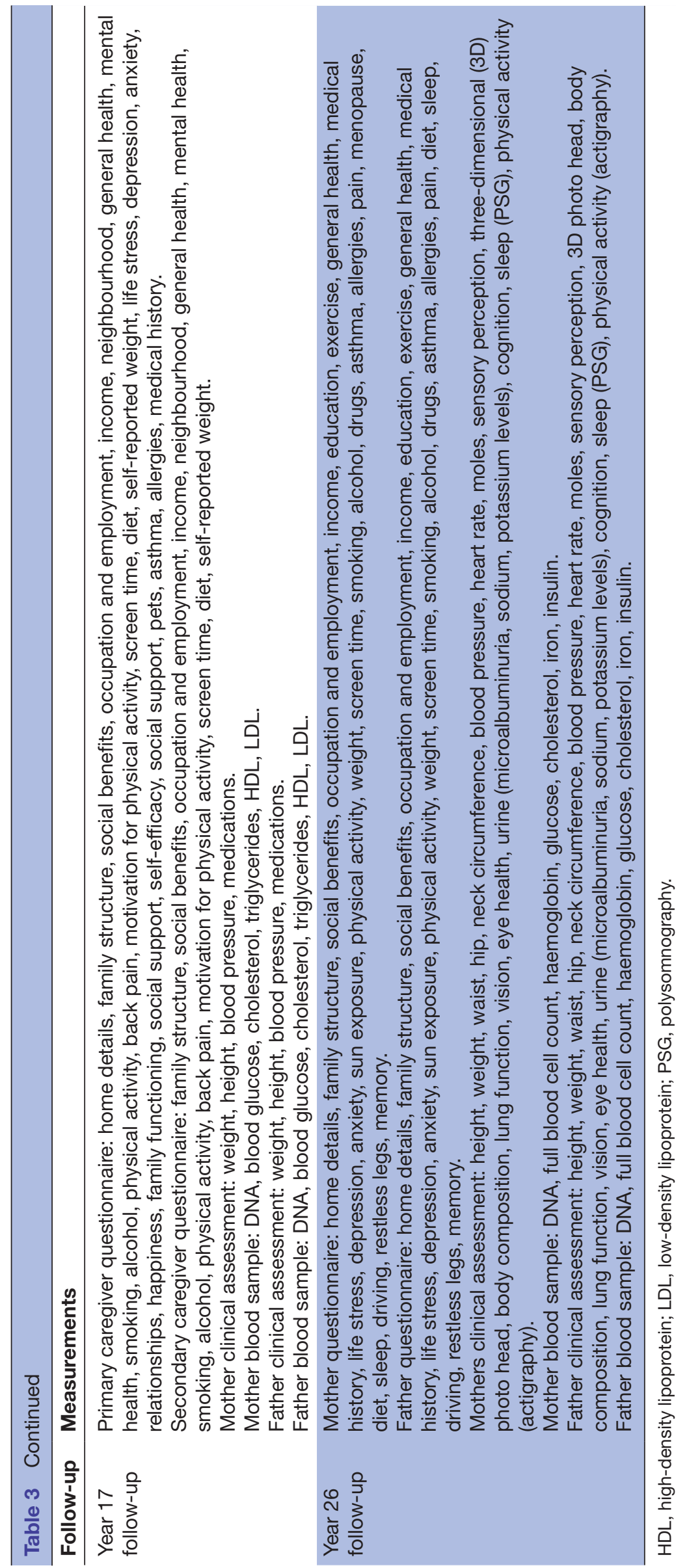


registrations, hospital admission records or mental health information systems, the Raine Study team is exploring multiple data linkage possibilities at the moment.

\section{Patient and public involvement}

The Raine Study values participant involvement in decisions about cohort assessments, activities and research projects. In the early years, Raine Study participants were not involved in designing the study, developing research questions and outcome measures, but this has changed over the years. Generation 2 participants have been involved through the Community Advisory Committee since they were approximately 10 years old. Since 2017 participants of both Generation 1 and 2 are involved in all levels of the Raine Study management. Representatives of each generation are present at the management meetings and play an important role in providing advice and feedback on proposed studies. They ensure that research protocols are acceptable from a participant's point of view.

\section{Findings to date}

Until the year 26 follow-up of Generation 1, the focus of research within the Raine Study has been on outcomes for Generation 2, with information on the parents being used to examine its influence on their children's outcomes. Some of those key findings and publications relating to the Generation 1 cohort are described below. Analyses of data from the recent Generation 1 year 26 follow-up are underway and findings will be presented in future papers that specifically focus on this generation.

\section{Genetics}

Raine Study data were used in a genome-wide association study aimed at identifying maternal genetic variants associated with offspring's birth weight. A few associations were found, and indicated effects of maternal glucose, cytochrome P450 activity, gestational duration, maternal blood pressure and immune function on fetal growth. ${ }^{5}$

\section{Cardiometabolic}

Recent findings showed that several characteristics of mothers, such as obesity, early mid-gestational weight gain and area-level socioeconomic status were associated with non-alcoholic fatty liver disease in offspring. ${ }^{6}$ body mass index (BMI) and weight gain of mothers before and in early pregnancy has been found to be associated with adiposity and cardiometabolic characteristics in their adolescent children. ${ }^{7}$ Furthermore, maternal stress in pregnancy has been shown to be associated with lower blood pressure and lower odds of hypertension in their children when they were aged 20 years. ${ }^{8}$

\section{Respiratory, immunology, inflammation}

In utero smoke exposure, a family history of asthma and maternal hypertension during pregnancy was found to be associated with reduced offspring respiratory function after birth, leading to speculation that these factors adversely affect lung development in utero. ${ }^{9}$ Also, smoking during pregnancy was shown to increase the risk of respiratory problems such as asthma and wheezing in offspring, up until adolescence, however these increased risks appear to be independent of the effects on lung function and immune function. ${ }^{10}$

\section{Hormonal and reproduction}

Maternal androgens did not appear to contribute to polycystic ovary syndrome in offspring. ${ }^{11}$ Specifically, maternal androgen levels, measured at 18 and 24 weeks pregnancy, were not significantly related to offspring ovarian morphology measured by transabdominal ultrasound examinations. ${ }^{11}$ Also, testosterone levels of pregnant women were found to be significantly correlated to ovarian function in their adolescent daughters, as measured by early follicular-phase circulating anti-Müllerian hormone levels. ${ }^{12}$

\section{Musculoskeletal}

Parents with back pain were more likely to have offspring who experienced back pain, with the ORs increasing from 1.41 (95\% CI 1.13 to 177) if one parent had back pain to 1.61 (95\% CI 1.07 to 2.48) if both parents had back pain. ${ }^{13}$

\section{Psychological}

Several maternal factors in the antenatal, perinatal and postnatal period have been shown to be risk factors for behavioural problems in offspring at age 2 and age $5 .{ }^{14}$ For example, the ORs for behaviour problems at age 2 and age 5 were 1.20 (95\% CI 1.06 to 1.37 ) and 1.17 (95\% CI 1.08 to 1.27$)$, respectively, when the mother experienced multiple stress events during pregnancy. ${ }^{14}$ Also, maternal experience of several stress events during pregnancy was found to be related to the offspring's later risk for behavioural problems up until at least 14 years of age. The nature of the stress events did not seem to matter, as both independent (eg, death of a relative, job loss) and dependent (eg, financial problems, marital problems) stressors were found to be significant predictors of behaviour problems in offspring. ${ }^{15}$

\section{Biological}

Maternal vitamin D concentrations were not related to autism spectrum disorder in offspring. ${ }^{16}$ Specifically, maternal vitamin $\mathrm{D}$ was found to be unrelated to most scales of the Autism-Spectrum Quotient, except the Attention Switching subscale, although low maternal vitamin $\mathrm{D}$ were found to be related to an increased risk their offspring scoring high on the Attention Switching subscale. ${ }^{16}$ A related study found that low maternal vitamin $\mathrm{D}$ concentrations were associated with lower peak bone mass in offspring at 20 years of age. ${ }^{17}$

\section{Diet}

Raine Study data contributed to a large study which combined data from multiple cohorts with the purpose of examining infant feeding and growth trajectory patterns in childhood and body composition in young 
adulthood. ${ }^{18}$ The study showed that maternal education was positively associated with a persistent, rapid, accelerating growth trajectory of the offspring (OR 3.12; 95\% CI 1.62 to 6.03 ) but not with an early, non-persistent, rapid growth trajectory. The study also showed that an increase in maternal prepregnancy BMI or maternal smoking during pregnancy increased the odds of offspring developing a rapid-growth pattern compared with a normative-growth pattern.

\section{Perinatal and life-course environmental exposures}

Maternal adverse life events occurring during pregnancy such as separation, divorce, marital problems, pregnancy problems, job loss, financial problems or death of a close relative were found to increase the risk for atopic disorders, asthma and eczema in offspring. ${ }^{19}$

\section{Education and work}

Non-linear and dynamic relationships were found between maternal working hours and offspring overweight or obesity. ${ }^{20}$ Specifically, young children (aged 2-5 years) were found to be less likely to be overweight or obese when mothers worked 24 hours or less per week, in comparison to when mothers worked full time ( 35 hours or more). Older children (aged 8-14 years) were more likely to be overweight or obese when their mother worked $<35$ hours per week or $>41$ hours per week, compared with when mothers worked 35-40 hours per week. This study also showed that up to a certain number of hours, maternal employment is beneficial for children's body weight. ${ }^{20}$ Stress events experienced by pregnant women were also shown to be related to their offspring's school performance, but the effects were different for boys and girls. ${ }^{21}$ For example, four or more stressful events were associated with lower reading scores in girls, while three or more stressful events were associated with higher reading scores in boys. ${ }^{21}$

\section{Strengths and limitations}

The strengths of the Raine Study include its longitudinal study design, the large amount and variety of data collected over 27 years and the inclusion of multiple generations from which data have been collected. A substantial number of investigators with a strong multidisciplinary focus are working with the data and form multiple (inter) national collaborations. These strengths, together with a clear governance structure and research administration procedures, ensures high-quality output and clinical relevance of Raine Study findings. Another strength of the Raine Study is its active engagement by participants in all levels of the organisation, which helps maintain the retention rate as high as possible.

Weaknesses of all generations of the Raine Study include its homogeneity in regard to the ethnicity of its participants, the moderate sample size and the gradual decline in the number of participants. ${ }^{3}$ Important weaknesses of the Generation 1 cohort relate to the relative inconsistency of the data across the follow-ups, as not all variables were collected at every follow-up, to variability in respondents (mostly mothers, but also fathers, primary caregivers, secondary caregivers, etc), meaning that data of all mothers and fathers are not available at each follow-up. However, the Raine Study is committed to future follow-up of Generation 1, which will improve the consistency in variables and respondents and thereby benefit further longitudinal data analyses.

Acknowledgements The authors would like to thank the Raine Study participants and their families for their ongoing participation in the study and the Raine Study staff for their dedicated commitment to co-ordination and data collection. The authors would also like to thank Angela Jacques for her assistance with the analyses.

Contributors MLD wrote the manuscript and performed the analyses, PE and LS are the (scientific) directors of the Raine Study and contributed significantly to the design and acquisition of the Raine Study and revised this manuscript critically. All authors agree to be accountable for all aspects of this work.

Funding The Raine Study receives core funding support from The University of Western Australia, Curtin University, Telethon Kids Institute, Women and Infants Research Foundation, Edith Cowan University, Murdoch University, The University of Notre Dame Australia and the Raine Medical Research Foundation. Funding of data collection and processing of data has been provided by the National Health and Medical Research Council of Australia (\#880441, 930745, 963209, 211912, 003209, 32300, 403981, 353514, 458623, 403968, 572613, 1084947, 1080492), and in chronological order: King Edward Memorial Hospital Research Foundation, Raine Medical Research Foundation, Glaxo Wellcome, The Asthma Foundation of Western Australia, Healthway (\#6006, 14123), Telstra Foundation, Cardiovascular Lipid Pfizer Grant, Australian Arthritis Foundation, The Stanley Trust (UK), Ada Bartholomew Medical Research Trust, Gastroenterology Society of Australia, Fremantle Hospital Medical Research Foundation, Women and Infants Research Foundation, Rotary Health Research, Canadian Institutes of Health Research, National Heart Foundation, Channel 7 Telethon Trust, Princess Margaret Hospital Foundation, Dairy Health and Nutrition Consortium, Danish Council for Strategic Studies, Smarttots, Asthma Foundation, Western Australia Department of Health Future Health Fund, Western Australia Department of Health Targeted Research Fund. Storage of biosamples has been enabled by substantial in-kind support from King Edward Memorial Hospital, Telethon Kids Institute and Royal Perth Hospital.

Competing interests None declared.

Patient consent for publication Not required.

Provenance and peer review Not commissioned; externally peer reviewed.

Data sharing statement The Raine Study encourages collaboration with national and international researchers. More details about the Raine Study, data and how to get access to the data are published on the website of the Raine Study (www. rainestudy.org.au).

Open access This is an open access article distributed in accordance with the Creative Commons Attribution Non Commercial (CC BY-NC 4.0) license, which permits others to distribute, remix, adapt, build upon this work non-commercially, and license their derivative works on different terms, provided the original work is properly cited, appropriate credit is given, any changes made indicated, and the use is non-commercial. See: http://creativecommons.org/licenses/by-nc/4.0/.

\section{REFERENCES}

1. Newnham JP, Reid SE. A randomised controlled trial of doppler waveform analysis in obstetrics. 1987.

2. Newnham JP, Michael CA, Landau LI, et al. The Origins of Disease in the Fetus, the Child and the Young Adult: A Western Australian Pregnancy Cohort Study. 1988.

3. Straker L, Mountain J, Jacques A, et al. Cohort Profile: The Western Australian Pregnancy Cohort (Raine) Study-Generation 2. Int J Epidemiol 2017;46:1384-1385j.

4. Newnham JP, Evans SF, Michael CA, et al. Effects of frequent ultrasound during pregnancy: a randomised controlled trial. Lancet 1993;342:887-91.

5. Beaumont RN, Warrington NM, Cavadino A, et al. Genome-wide association study of offspring birth weight in 86577 women identifies 
five novel loci and highlights maternal genetic effects that are independent of fetal genetics. Hum Mol Genet 2018;27:742-56.

6. Ayonrinde OT, Adams LA, Mori TA, et al. Sex differences between parental pregnancy characteristics and nonalcoholic fatty liver disease in adolescents. Hepatology 2018;67.

7. Gaillard R, Welten M, Oddy WH, et al. Associations of maternal prepregnancy body mass index and gestational weight gain with cardio-metabolic risk factors in adolescent offspring: a prospective cohort study. BJOG 2016;123:207-16.

8. Bhat SK, Beilin LJ, Robinson M, et al. Contrasting effects of prenatal life stress on blood pressure and body mass index in young adults. $J$ Hypertens 2015;33:711-9.

9. Stick SM, Burton PR, Gurrin L, et al. Effects of maternal smoking during pregnancy and a family history of asthma on respiratory function in newborn infants. Lancet 1996;348:1060-4.

10. Hollams EM, de Klerk NH, Holt PG, et al. Persistent effects of maternal smoking during pregnancy on lung function and asthma in adolescents. Am J Respir Crit Care Med 2014;189:401-7.

11. Hickey M, Sloboda DM, Atkinson HC, et al. The relationship between maternal and umbilical cord androgen levels and polycystic ovary syndrome in adolescence: a prospective cohort study. J Clin Endocrinol Metab 2009;94:3714-20.

12. Hart R, Sloboda DM, Doherty DA, et al. Circulating maternal testosterone concentrations at 18 weeks of gestation predict circulating levels of antimüllerian hormone in adolescence: a prospective cohort study. Fertil Steril 2010;94:1544-7.

13. O'Sullivan PB, Straker LM, Smith A, et al. Carer experience of back pain is associated with adolescent back pain experience even when controlling for other carer and family factors. Clin J Pain 2008;24:226-31.

14. Robinson M, Oddy $\mathrm{WH}, \mathrm{Li} \mathrm{J}$, et al. Pre- and postnatal influences on preschool mental health: a large-scale cohort study. J Child Psychol Psychiatry 2008;49:1118-28.

15. Robinson M, Mattes E, Oddy WH, et al. Prenatal stress and risk of behavioral morbidity from age 2 to 14 years: the influence of the number, type, and timing of stressful life events. Dev Psychopathol 2011;23:507-20.

16. Whitehouse AJ, Holt BJ, Serralha M, et al. Maternal vitamin D levels and the autism phenotype among offspring. $J$ Autism Dev Disord 2013;43:1495-504.

17. Zhu K, Whitehouse AJ, Hart PH, et al. Maternal vitamin D status during pregnancy and bone mass in offspring at 20 years of age: a prospective cohort study. J Bone Miner Res 2014;29:1088-95.

18. Rzehak P, Oddy $\mathrm{WH}$, Mearin ML, et al. Infant feeding and growth trajectory patterns in childhood and body composition in young adulthood. Am J Clin Nutr 2017:106:568-80.

19. Hartwig IR, Sly PD, Schmidt LA, et al. Prenatal adverse life events increase the risk for atopic diseases in children, which is enhanced in the absence of a maternal atopic predisposition. $J$ Allergy Clin Immunol 2014;134:160-9.

20. Li J, Akaliyski P, Schäfer J, et al. Non-linear relationship between maternal work hours and child body weight: Evidence from the Western Australian Pregnancy Cohort (Raine) Study. Soc Sci Med 2017:186:52-60.

21. Li J, Robinson M, Malacova E, et al. Maternal life stress events in pregnancy link to children's school achievement at age 10 years. $J$ Pediatr 2013;162:483-9. 